\title{
Characterizations of Restricted Pairs of Planar Graphs Allowing Simultaneous Embedding with Fixed Edges
}

\author{
J. Joseph Fowler ${ }^{1}$, Michael Jünger ${ }^{2}$, Stephen Kobourov ${ }^{1}$, and Michael Schulz ${ }^{2}$ \\ 1 University of Arizona, USA \{jfowler, kobourov\}@cs.arizona.edu * \\ ${ }^{2}$ University of Cologne, Germany \{mjuenger, schulz\}@informatik. uni-koeln.de *夫
}

\begin{abstract}
A set of planar graphs share a simultaneous embedding if they can be drawn on the same vertex set $V$ in the Euclidean plane without crossings between edges of the same graph. Fixed edges are common edges between graphs that share the same simple curve in the simultaneous drawing. Determining in polynomial time which pairs of graphs share a simultaneous embedding with fixed edges (SEFE) has been open.

We give a necessary and sufficient condition for whether a SEFE exists for pairs of graphs whose union is homeomorphic to $K_{5}$ or $K_{3,3}$. This allows us to characterize the class of planar graphs that always have a SEFE with any other planar graph. We also characterize the class of biconnected outerplanar graphs that always have a SEFE with any other outerplanar graph. In both cases, we provide efficient algorithms to compute a SEFE. Finally, we provide a linear-time decision algorithm for deciding whether a pair of biconnected outerplanar graphs has a SEFE.
\end{abstract}

\section{Introduction}

In many practical applications including the visualization of large graphs and very-large-scale integration (VLSI) of circuits on the same chip, edge crossings are undesirable. A single vertex set can be used with multiple edge sets that each correspond to different edge colors or circuit layers. While the pairwise union of all edge sets may be non-planar, a planar drawing of each layer may be possible, as crossings between edges of distinct edge sets are permitted. Finding such drawings is the basic problem of simultaneous embedding (SE) and this can be viewed as a generalization of the notion of planarity to multiple graphs.

Without restrictions on the types of edges, any number of planar graphs can be drawn on the same fixed set of vertex locations [13]. However, difficulties arise once straight-line edges are required. This is the problem of simultaneous geometric embedding (SGE). If edge bends are allowed, then having common edges drawn in the same way using the same simple curve preserves the "mental map". Such edges are called fixed edges leading to the problem of simultaneous embedding with fixed edges (SEFE). Since straight-line edges between a pair of vertices are also fixed edges, any graph that has a SGE also has a SEFE, but the converse is not true; see Fig. 1 that shows SGE $\subset$ SEFE $\subset$ SE.

\footnotetext{
* This work is supported in part by NSF grants CCF-0545743 and ACR-0222920.

** This work is supported in part by the German Science Foundation (JU204/11-1) and by the German Academic Exchange Service.
} 


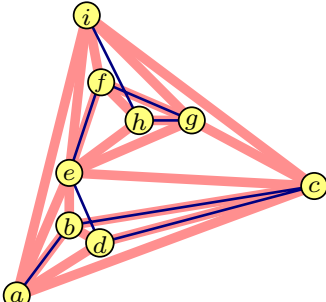

(a)

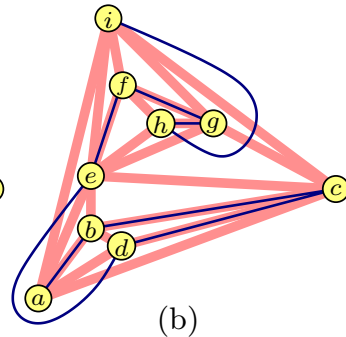

(b)

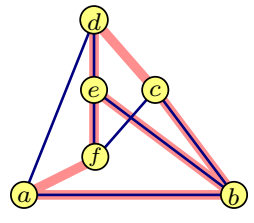

(c)

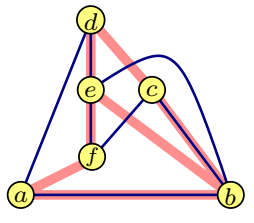

(d)

Fig. 1. The path and planar graph in (a) do not have a SGE with straight-line edges [2], but have a SEFE in (b). The two outerplanar graphs in (c) do not have a SEFE, but have a SE in (d) if the edge $(b, e)$ is not fixed.

Deciding whether two graphs have a SGE is NP-hard [6], while deciding whether three graphs have a SEFE is NP-complete [9]. However, deciding whether two graphs have a SEFE in polynomial-time remains open. We give a necessary condition in terms of forbidden minors for when pairs of graphs can have a SEFE. This leads to a polynomial-time decision algorithm in the restricted case of pairs of biconnected outerplanar graphs. We also characterize the class of biconnected outerplanar graphs that always have a SEFE with any other outerplanar graph. Finally, we characterize the graphs that always have a SEFE with any planar graph and compute a SEFE when possible.

\subsection{Related Work}

Any number of stars, two caterpillars (trees whose removal of all leaves gives a path) and two cycles always have a SGE, whereas three paths and two trees may not $[2,10]$. Which graphs always have a SGE with a path, a caterpillar, a tree, or a cycle remains unknown. For the case of SEFE, a planar graph and a tree always have a SEFE, whereas two outerplanar graphs do not [8]. This shows that the topological problem of SEFE is less restricted than the geometric problem of SGE. Note that this is unlike standard planarity where the sets of topological and geometric planar graphs are identical [5]. Planar graphs are characterized in terms of the forbidden graphs, $K_{5}$ and $K_{3,3}$, which form two minimum examples of non-planarity $[12,14]$. No similar characterization for SEFE in terms forbidden pairs has been given until now, even for restricted pairs of planar graphs.

\subsection{Our Contribution}

1. We show there exist three paths without a SEFE. We provide a necessary and sufficient condition in terms of 17 minimal forbidden pairs for when a pair of graphs whose union forms a subdivided $K_{5}$ or $K_{3,3}$ has a SEFE.

2. Using this condition, we characterize the class of planar graphs that have a SEFE with any planar graph to be the set of (i) forests, (ii) circular caterpillars, (iii) $K_{4}$, and (iv) subgraphs of $K_{3}$-multiedges; see Fig. 2 (a)-(d). We efficiently compute a SEFE in each case. We show that any other graph not in this class contains a subgraph homeomorphic to a cycle and a disjoint edge. We provide a similar characterization for the class of biconnected outerplanar graphs that always share a SEFE with any outerplanar graph; see Fig. 2(e). Table 1 summarizes our results. 


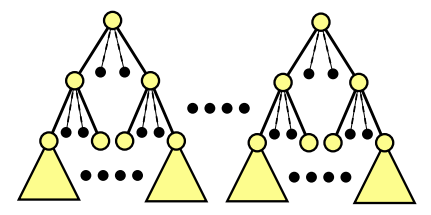

(a)

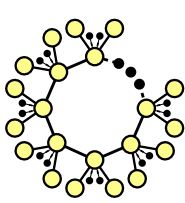

(b)

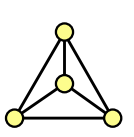

(c)

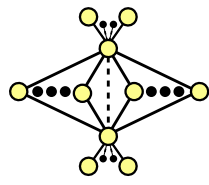

(d)

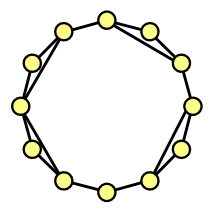

(e)

Fig. 2. Forests in (a), circular caterpillars (removal of all degree-1 vertices yields a cycle) in (b), $K_{4}$ in (c) and subgraphs of $K_{3}$-multiedges (an edge with any number of incident edges) in (d) have a SEFE with any planar graph. $K_{3}$-cycles ( $n$-cycles with chords that form 3-cycles with the $n$-cycles) as in (e) have a SEFE with any outerplanar graph.

3. We determine which pairs of biconnected outerplanar graphs can have a SEFE using a forbidden outerplanar pair. This leads to a linear-time decision algorithm for this restricted case.

\subsection{Preliminaries}

Let $P$ be a set of $n$ distinct points in the plane $\mathbb{R}^{2}$. A planar drawing of $G(V, E)$ with $|V|=n$ on $P$ consists of a bijection $\sigma: V \rightarrow P$ with a simple curve for each edge $(u, v) \in E$ drawn in the plane $\mathbb{R}^{2}$ connecting the points $\sigma(u)$ and $\sigma(v)$ with curves that only intersect at endpoints. Let $\mathcal{G}=\left\{G_{1}\left(V, E_{1}\right), G_{2}\left(V, E_{2}\right), \ldots\right.$, $\left.G_{k}\left(V, E_{k}\right)\right\} . \mathcal{G}$ has a simultaneous embedding (SE) if there exist planar drawings of $G_{i}\left(V, E_{i}\right)$ with the same bijection $\sigma: V \rightarrow P$. If each edge is a straightline segment, then $\mathcal{G}$ has a simultaneous geometric embedding (SGE). If every common edge in $\mathcal{G}$ connecting a pair of vertices uses the same simple curve, then $\mathcal{G}$ has a simultaneous embedding with fixed edges (SEFE).

In a graph $G(V, E)$, subdividing an edge $(u, v) \in E$ replaces edge $(u, v)$ with the pair of edges $(u, w)$ and $(w, v)$ in $E$ by adding $w$ to $V$. A subdivision of $G$ is obtained through a series of edge subdivisions. Contraction of edge $(u, v)$ replaces the vertices $u$ and $v$ with the vertex $w$ that is adjacent to all the vertices that were adjacent to either $u$ or $v$. A minor $H$ of $G$ is obtained through a series of edge contractions and edge deletions. A graph $G(V, E)$ is isomorphic to a graph $\tilde{G}(\tilde{V}, \tilde{E})$ if there exists a bijection $f: V \rightarrow \tilde{V}$ such that $(u, v) \in E$ if and only if $(f(u), f(v)) \in \tilde{E}$. A graph $G(V, E)$ is homeomorphic to a graph $\tilde{G}(\tilde{V}, \tilde{E})$ if the subdivisions of $G$ and $\tilde{G}$ are isomorphic.

\begin{tabular}{||c|c|c||c|c|c|c|c||}
\cline { 2 - 8 } \multicolumn{1}{c|}{} & \multicolumn{2}{c|}{ SGE } & \multicolumn{5}{c||}{ SEFE } \\
\cline { 2 - 8 } \multicolumn{1}{c|}{} & Path & Tree & Forest & $\begin{array}{c}\text { Circular } \\
\text { caterpillar }\end{array}$ & $K_{4}$ & $\begin{array}{c}K_{3^{-}} \\
\text {multiedge }\end{array}$ & $\begin{array}{c}K_{3^{-}} \\
\text {cycle }\end{array}$ \\
\hline \hline Path & $\checkmark[2]$ & $?$ & $\checkmark[8]$ & $\checkmark[8]$ & $\checkmark[8]$ & $\checkmark[8]$ & $\checkmark[8]$ \\
\hline Caterpillar & $\checkmark[2]$ & $?$ & $\checkmark[8]$ & $\checkmark[8]$ & $\checkmark[8]$ & $\checkmark[8]$ & $\checkmark[8]$ \\
\hline Tree & $?$ & $\boldsymbol{X}[10]$ & $\checkmark[8]$ & $\checkmark[8]$ & $\checkmark[8]$ & $\checkmark[8]$ & $\checkmark[8]$ \\
\hline Outerplanar & $?$ & $\boldsymbol{X}[10]$ & $\checkmark$ & $\checkmark$ & $\checkmark$ & $\checkmark$ & $\checkmark$ \\
\hline Planar & $\boldsymbol{X}[2]$ & $\boldsymbol{X}[2,10]$ & $\checkmark$ & $\checkmark$ & $\checkmark$ & $\checkmark$ & $\boldsymbol{X}$ \\
\hline
\end{tabular}

Table 1. Old and new results for SGE and SEFE pairs. The shaded pairs are new. 


\section{Forbidden Simultaneous Embeddings with Fixed Edges}

We begin with Kuratowski's and Wagner's planar graph theorems $[12,14]$.

Theorem 1 (Kuratowski, Wagner) A graph is non-planar if and only if it has a subgraph homeomorphic to $K_{5}$ or $K_{3,3}$ or has $K_{5}$ or $K_{3,3}$ as a minor.

\subsection{Forbidden Triples of Paths and Cycles}

Next we show that the triples without a SGE of three paths in [2] and three cycles in [1] extend to the case of SEFE.

Theorem 2 There exist three paths on 9 vertices and three cycles on 6 vertices without a SEFE.

Proof. Consider the three paths $g-d-h-c-e-a-f-b-i, h-d-i-b-e-c-f-a-g$, and $i-d-g-a-e-b-f-c-h$ and the three cycles $a-d-c-f-b-e-a, a-e-c-d-b-f-a$, and $a-f-c-e-b-d-a$ shown in Fig. 3. In both cases, the union forms a subdivided $K_{3,3}$ and any drawing must have a crossing by Theorem 1 . Each edge in the union belongs to two paths (or two cycles). Such a crossing must be between two pairs of paths (or cycles). Since there are only three paths (or three cycles) and fixed edges are being used, one path (or cycle) must self-intersect.

\subsection{Minimal Forbidden Pairs}

Suppose a pair of graphs $G_{1}\left(V, E_{1}\right)$ and $G_{2}\left(V, E_{2}\right)$ does not have a SEFE as in Fig. 4(a). If deleting any edge from either graph allows a SEFE, then $G_{1}$ and $G_{2}$ are edge minimal as in Fig. 4(b). If a degree-2 vertex $v$ (adjacent to $u$ and $w$ ) in the union of $G_{1}$ and $G_{2}$ is not a degree- 1 vertex in either $G_{1}$ or $G_{2}$, then we can unsubdivide the vertex by deleting $v$ and replacing edges $(u, v)$ and $(v, w)$ with the edge $(u, w)$ in $G_{1}$ and/or $G_{2}$. A pair of graphs for which this can no longer be done is vertex minimal as in Fig. 4(c). A minimal forbidden pair does not have a SEFE and is edge and vertex minimal.

We define the union $G_{1} \cup G_{2}$ and the intersection $G_{1} \cap G_{2}$ as having edge sets $E_{1} \cup E_{2}$ and $E_{1} \cap E_{2}$, respectively; see Fig. 4(c)-(d). Suppose then that $G_{1} \cup G_{2}$ is homeomorphic to a graph $G$ with no degree-2 vertices. Let $u \rightsquigarrow v$ in $G_{1} \cup G_{2}$ be the path corresponding to the subdivided edge $(u, v)$ in $G$. Path $u \rightsquigarrow v$ is incident to $x \rightsquigarrow y$ in $G_{1} \cup G_{2}$ if and only if $(u, v)$ is incident to $(x, y)$ in $G$. An alternating edge is a $u \rightsquigarrow v$ path in which the edges strictly alternate between being in either $G_{1}$ or $G_{2}$; see Fig. 4(e). An exclusive edge is a $u \rightsquigarrow v$ path composed of the edge $(u, v)$ that is only in $G_{1}$ or $G_{2}$; see Fig. 4(f)-(g), while an inclusive edge is composed of the fixed edge $(u, v)$ in $G_{1} \cap G_{2}$; see Fig. 4(d).

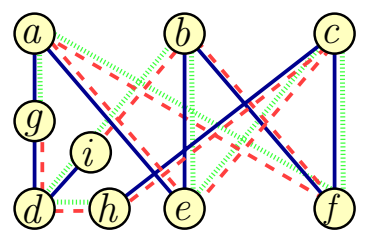

(a) 3 paths on 9 vertices

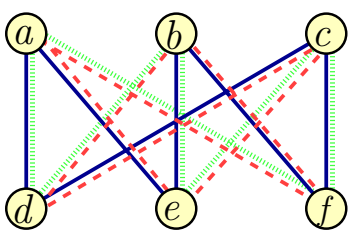

(b) 3 cycles on 6 vertices

Fig. 3. Two graph triples without a SEFE. 


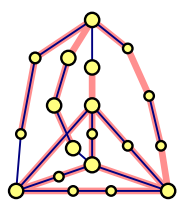

(a)

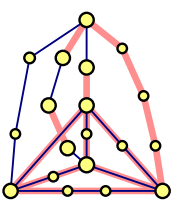

(b)

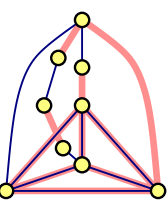

$G_{1} \cup G_{2}$

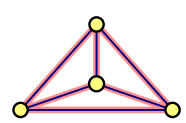

(d)

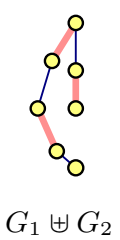

(e)

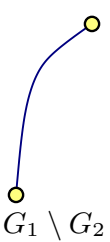

(f)

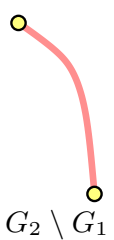

(g)

Fig. 4. Removing extraneous edges from (a) gives (b). Unsubdividing degree-2 vertices in (b) gives (c) that can be partitioned into the four subgraphs in $(d)-(g)$.

Lemma 3 Any pair of graphs $G_{1}\left(V, E_{1}\right)$ and $G_{2}\left(V, E_{2}\right)$ can be reduced to a pair in which every $u \rightsquigarrow v$ path is either an inclusive, exclusive, or alternating edge. Proof. We examine each $u \rightsquigarrow v$ path $p$ in $G_{1} \cup G_{2}$. If path $p$ is in $G_{1} \cap G_{2}$, we replace $p$ with a single inclusive edge $(u, v)$ in both $G_{1}$ and $G_{2}$. If $p$ is in $G_{i}$ but is missing edges in $G_{j}$ for $i \neq j$, we replace it with the single exclusive edge $(u, v)$ in $G_{i}$. If $p$ is missing an edge from each graph, we make $p$ into an alternating edge by deleting edges from $p$ in either $G_{1}$ or $G_{2}$ until each edge along $p$ is no longer in $G_{1} \cap G_{2}$. Then we unsubdivide $p$ until it is strictly alternating. We can always avoid crossings along edges of $u \rightsquigarrow v$ paths contained in $G_{1} \cap G_{2}$ reduced in this way. Hence, neither operation changes whether the pair has a SEFE.

Suppose $G_{1}$ and $G_{2}$ are a reduced pair, which is a pair of graphs where all $u \rightsquigarrow v$ paths have been reduced. The alternating edge subgraph, $G_{1} \uplus G_{2}$, is the subgraph of $G_{1} \cup G_{2}$ consisting only of alternating edges. The exclusive edge subgraph of $G_{1}, G_{1} \backslash G_{2}$, is the subgraph of $G_{1} \cup G_{2}$ consisting of exclusive edges from $G_{1}$, where $G_{2} \backslash G_{1}$ is defined analogously. Hence, edges of $G_{1} \cup G_{2}$ are partitioned into $G_{1} \cap G_{2}, G_{1} \uplus G_{2}, G_{1} \backslash G_{2}$, and $G_{2} \backslash G_{1}$; see Fig. 4(d)-(g). Next we see why we only need to consider crossings between non-incident edges.

Observation 4 Crossings between incident edges in a non-planar drawing can be removed without affecting the number of crossings of non-incident edges.

This can be done by swapping the simple curves from the incident vertex to the first intersection point $p$. Separating the curves at $p$ by a small distance eliminates the crossing without affecting the rest of the drawing. Repeating this process removes all crossings of incident edges. Hence, we only need to consider crossings of non-incident edges in a simultaneous drawing with fixed edges. Removing an edge from either $K_{5}$ or $K_{3,3}$ of Theorem 1, allows a planar embedding. Only one crossing needs to be introduced when replacing the edge, since there is at most one edge separating any pair of faces in the embedding. This fact along with Observation 4 gives the next corollary.

Corollary 5 (a) Every drawing of $K_{5}$ or $K_{3,3}$ has a crossing between nonincident edges. (b) $K_{5}$ or $K_{3,3}$ can be drawn with only one crossing between any pair of non-incident edges.

We use this corollary to produce a sufficient condition for SEFE.

Lemma 6 Suppose the union $G_{1} \cup G_{2}$ of a reduced pair $\left(G_{1}, G_{2}\right)$ is homeomorphic to $K_{5}$ or $K_{3,3}$. Let $u \rightsquigarrow v$ and $x \rightsquigarrow y$ be non-incident paths in $G_{1} \cup G_{2}$ but not in $G_{1} \cap G_{2}$. If either path belongs to $G_{1} \uplus G_{2}$ or one belongs to $G_{1} \backslash G_{2}$ and the other belongs to $G_{2} \backslash G_{1}$, then $G_{1}$ and $G_{2}$ have a SEFE 
Proof. By Corollary $5(\mathrm{~b})$, a $K_{5}$ or a $K_{3,3}$ can always be drawn so that only $(u, v)$ and $(x, y)$ cross. Hence, there is a SEFE in which an alternating edge in $G_{1} \uplus G_{2}$ only crosses an edge in either $G_{1} \backslash G_{2}$ or $G_{2} \backslash G_{1}$. Likewise, an edge in $G_{1} \backslash G_{2}$ can cross any non-incident edge in $G_{2} \backslash G_{1}$.

With Lemma 6 we determine when a $K_{5}$ or a $K_{3,3}$ pair has a SEFE.

Corollary 7 Suppose the union $G_{1} \cup G_{2}$ of a reduced pair $\left(G_{1}, G_{2}\right)$ is homeomorphic to $K_{5}$ or $K_{3,3}$. The pair $\left(G_{1}, G_{2}\right)$ has no SEFE if and only if (i) every non-incident edge of an alternating edge in $G_{1} \uplus G_{2}$ is in $G_{1} \cap G_{2}$ and (ii) every non-incident edge of an exclusive edge in $G_{1} \backslash G_{2}$ is in $G_{1}$.

Proof. For necessity, suppose the pair $\left(G_{1}, G_{2}\right)$ does not have a SEFE. Consider an $x \rightsquigarrow y$ path in $G_{1} \cup G_{2}$ that is non-incident to an alternating edge $u \rightsquigarrow v$ in $G_{1} \uplus G_{2}$ in which $x \rightsquigarrow y$ is not in $G_{1} \cap G_{2}$. By Lemma 6 , the pair $\left(G_{1}, G_{2}\right)$ would have a SEFE since $u \rightsquigarrow v$ is in $G_{1} \uplus G_{2}$ and neither path is in $G_{1} \cap G_{2}$. Next consider an $x \rightsquigarrow y$ path in $G_{1} \cup G_{2}$ that is non-incident to an exclusive edge $(u, v)$ in $G_{1} \backslash G_{2}$ in which $x \rightsquigarrow y$ is not in $G_{1}$. By Lemma 6 , the pair $\left(G_{1}, G_{2}\right)$ again would have a SEFE since $x \rightsquigarrow y$ either is in $G_{1} \uplus G_{2}$ or is in $G_{2} \backslash G_{1}$.

For sufficiency, suppose conditions (i) and (ii) hold. Since the union forms a subdivided $K_{5}$ or $K_{3,3}$, by Corollary 5 (a) at least one pair of non-incident paths $u \rightsquigarrow v$ and $x \rightsquigarrow y$ cross. If either is in $G_{1} \cap G_{2}$, then there must be a crossing in $G_{1}$ or $G_{2}$. If either is in $G_{1} \uplus G_{2}$, then by (i) the other would be in $G_{1} \cap G_{2}$, again giving a crossing in $G_{1}$ or $G_{2}$. If both are in $G_{i} \backslash G_{j}$ for $i \neq j$, then there is a crossing in $G_{i}$. Finally, (ii) prevents one edge being in $G_{1} \backslash G_{2}$ and the other edge being in $G_{2} \backslash G_{1}$. Hence, $G_{1}$ and $G_{2}$ do not have a SEFE.

Theorem 8 There are 17 minimal forbidden pairs with a union homeomorphic to $K_{5}$ or $K_{3,3}$.

Proof. Let $G_{i, j}$ denote the 17 pairs of graphs for $i \in\{1, \ldots, 17\}$ and $j \in\{1,2\}$ in Figs. 5 and 6 . One can verify that all the non-incident edges of any alternating edge are in the intersection and every edge non-incident to an exclusive edge of $G_{i, 1}$ is also in $G_{i, 1}$. This satisfies Corollary 7 implying that none of these pairs has a SEFE. Removing any edge means either (i) the union no longer forms a $K_{5}$

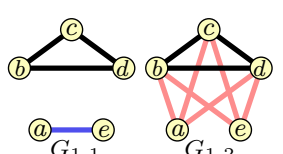

(a)

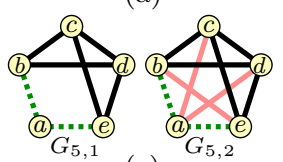

(e)

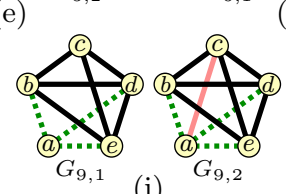

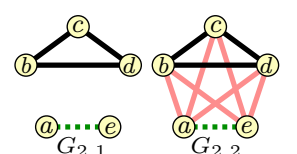

(b)

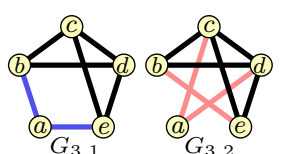

(c)

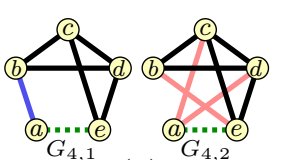

(d)

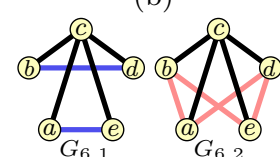

(f)

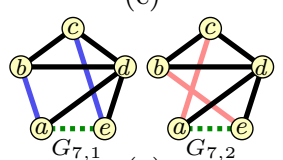

(g)

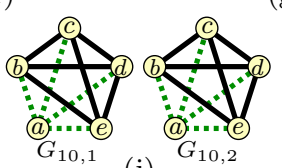

(j)

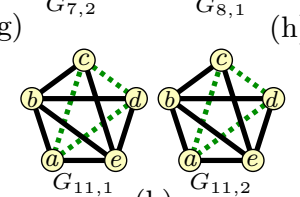

(k)

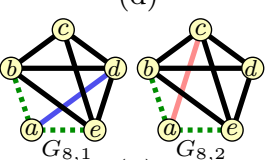

(h)

Fig. 5. Eleven $K_{5}$ minimal forbidden pairs. 


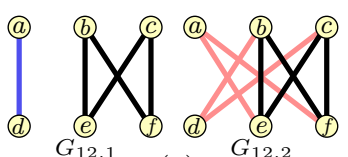

(a)

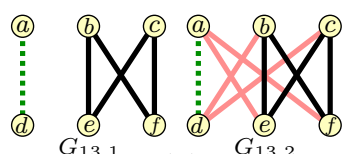

(b)

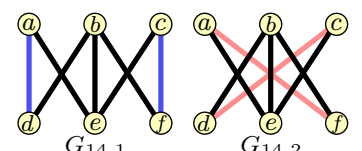

(c)

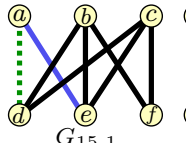

(d)

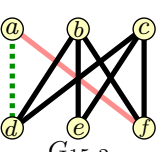

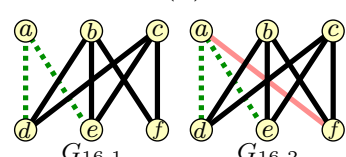

(e)

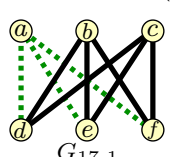

(f)

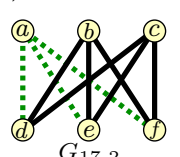

Fig. 6. Six $K_{3,3}$ minimal forbidden pairs.

or a $K_{3,3}$ or (ii) the intersection does not contain all the non-incident edges of $G_{i, 1} \uplus G_{i, 2}$ or of $G_{i, 1} \backslash G_{i, 2}$ (other than those already in $G_{i, 1}$ ) so that Corollary 7 is no longer satisfied. This implies that all 17 forbidden pairs are minimal.

We next show that our 17 pairs are the only minimal forbidden pairs homeomorphic to $K_{5}$ or $K_{3,3}$. Assume w.l.o.g. $\left(G_{1}, G_{2}\right)$ is a reduced minimal forbidden pair whose union forms a $K_{5}$ or a $K_{3,3}$ where $G_{2}$ has at least as many edges as $G_{1}$. We consider all the possibilities for edges to be in $G_{1} \backslash G_{2}$ or $G_{1} \uplus G_{2}$.

Pairs $\left(G_{1,1}, G_{1,2}\right),\left(G_{2,1}, G_{2,2}\right),\left(G_{12,1}, G_{12,2}\right)$, and $\left(G_{13,1}, G_{13,2}\right)$ are the only possibilities in which there is one exclusive edge in $G_{1}$ or one alternating edge in $G_{1} \uplus G_{2}$. Two non-incident alternating edges would violate Corollary 7 . The other case of two non-incident edges that are exclusive in $G_{1}$ is given by pairs $\left(G_{6,1}, G_{6,2}\right)$ and $\left(G_{14,1}, G_{14,2}\right)$. Three non-incident edges are only possible in a $K_{3,3}$, but adding all of their non-incident edges implies that $G_{1}$ is a $K_{3,3}$.

For the case of $G_{1} \cup G_{2}$ homeomorphic to $K_{5}$, the pairs $\left(G_{3,1}, G_{3,2}\right),\left(G_{4,1}, G_{4,2}\right)$, and $\left(G_{5,1}, G_{5,2}\right)$ give the three possibilities of two incident edges that are exclusive and/or alternating. Two incident exclusive edges with a third exclusive or alternating edge cannot happen since $G_{3,1}$ has seven edges with two incident exclusive edges. Adding another exclusive or alternating edge along with its non-incident edge would imply that $\left|G_{2} \backslash G_{1}\right|=\left|G_{1} \cup G_{2}\right|-\left|G_{1}\right|-\left|G_{1} \backslash G_{2}\right|=10-7-2=1$. This contradicts our assumption of $G_{2}$ having at least as many edges as $G_{1}$.

Two non-incident exclusive edges with a third incident alternating edge is given by the pair $\left(G_{7,1}, G_{7,2}\right)$. Two or three alternating edges that are all incident with another exclusive or alternating edge are given by the pairs $\left(G_{8,1}, G_{8,2}\right)$, $\left(G_{9,1}, G_{9,2}\right)$ and $\left(G_{10,1}, G_{10,2}\right)$, respectively. The last possibility of three alternating edges that are only pairwise incident is given by pair $\left(G_{11,1}, G_{11,2}\right)$ in which all the non-incident edges of each alternating edge is in the intersection.

For the case of $G_{1} \cup G_{2}$ homeomorphic to $K_{3,3}$, if there are two incident exclusive and/or alternating edges, then the third incident $u \rightsquigarrow v$ edge in the union is the only edge that can be in $G_{2} \backslash G_{1}$. This is because edges non-incident to $u \rightsquigarrow v$ are also in $G_{1}$ implying that $G_{2} \backslash G_{1}$ can only contain the edge $(u, v)$. Hence, $\left|G_{1} \backslash G_{2}\right|<\left|G_{2} \backslash G_{1}\right|=1$. Pairs $\left(G_{15,1}, G_{15,2}\right)$ with one exclusive edge and one alternating edge and $\left(G_{16,1}, G_{16,2}\right)$ with two alternating edges are the only possibilities for two incident edges. However, $u \rightsquigarrow v$ could be an alternating edge. The pair $G_{16,2}$ already has one exclusive edge with two incident alternating edges. This leaves three alternating edges that are all incident given by pair $\left(G_{17,1}, G_{17,2}\right)$ as the final possibility. 


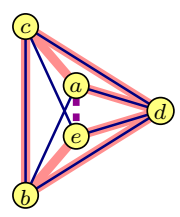

(a)

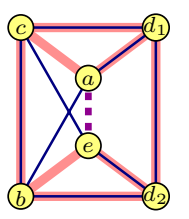

(b)

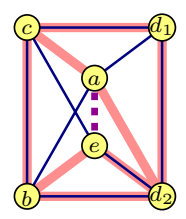

(c)

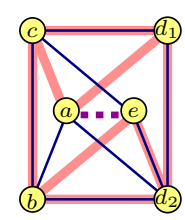

(d)

Fig. 7. The pair $\left(G_{7,1}, G_{7,2}\right)$ in (a) is a minor pair of the two forbidden pairs in (b) and (c), which have no SEFE, as well as the pair in (d), which has the given SEFE.

Unlike standard planar graphs in which the set of forbidden minors is identical to the set of forbidden subdivisions by Theorem 1, the same is not true for SEFE. Fig. 7 shows three pairs with the same minor pair $\left(G_{7,1}, G_{7,2}\right)$ in Fig. 7 (a). Each pair is obtained by "uncontracting" vertex $d$ to form the fixed edge $\left(d_{1}, d_{2}\right)$ in Figs. 7(b)-(d). Fig. 7(b)-(c) are forbidden pairs, whereas, Fig. 7(d) is not.

Figs. $7(\mathrm{c})-(\mathrm{d})$ are examples in which a new fixed edge $(a, d)$ is created from the exclusive edges $\left(a, d_{1}\right)$ in $G_{1} \backslash G_{2}$ and $\left(a, d_{2}\right)$ in $G_{2} \backslash G_{1}$ by contracting edge $\left(d_{1}, d_{2}\right)$ to vertex $d$ in Fig. $7(\mathrm{a})$. To avoid this, we define a fixed edge minor pair as a minor pair $\left(H_{1}, H_{2}\right)$ of $\left(G_{1}, G_{2}\right)$ that is obtained by only contracting edges in which no new fixed edges are created. Fig. 7(b) is an example in which Fig. 7(a) forms a fixed edge minor pair. This leads to the following corollary.

Corollary 9 Pair $\left(G_{1}, G_{2}\right)$ has no SEFE if the pair has a fixed edge minor pair $\left(H_{1}, H_{2}\right)$ isomorphic to one of the 17 minimal forbidden pairs of Theorem 8.

This forms a necessary condition for SEFE, but is insufficient since Fig. 7(c) does not have a SEFE, nor does it have any of the 17 fixed edge minor pairs.

\section{Characterizing SEFE with Planar Graphs}

We next determine the graphs that always have a SEFE with any planar graph and produce simultaneous drawings. Let $\mathcal{P}$ be the set of planar graphs and $\mathcal{P}_{\mathrm{SEFE}}$ be the subset of $\mathcal{P}$ containing forests, circular caterpillars (removal of all degree-1 vertices yields a cycle), $K_{4}$, and the subgraphs of $K_{3}$-multiedges (edge $(x, y)$ with the incident edges $(x, z)$ and/or $(y, z)$ for each $z \in V \backslash\{x, y\})$.

Lemma $10 G$ is in $\mathcal{P}_{\text {SEFE }}$ if and only if $G$ does not contain a subgraph homeomorphic to a $K_{3}$ and a disjoint edge.

Proof. First, we show necessity. Let $G \in \mathcal{P}_{\text {SEFE }}$ and let $H$ be the graph consisting of a $K_{3}$ and a disjoint edge. A forest has no cycles unlike $H$. While a circular caterpillar has a cycle, all the other edges are incident to the cycle. A $K_{4}$ has four vertices while $H$ has five. Finally, every subgraph of a $K_{3}$-multiedge with a cycle, either has a 3 -cycle, $x \rightsquigarrow y \rightsquigarrow z \rightsquigarrow x$, or a 4-cycle, $x \rightsquigarrow z_{1} \rightsquigarrow y \rightsquigarrow z_{2} \rightsquigarrow x$, if there is no edge $(x, y)$. In either case, every other edge is part of the cycle or is incident to $x$ or $y$

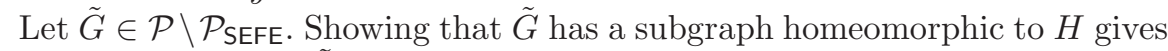
sufficiency. The graph $\tilde{G}$ must have a cycle since otherwise it would be a forest. Let $C$ be a cycle in $\tilde{G}$ of maximum length, and let $e$ be any edge in $\tilde{G} \backslash C$. Either 
the edge $e$ is incident to $C$ or the graph $\tilde{G}$ contains a subgraph homeomorphic to $H$. If the edge $e$ forms a chord of $C$ where $C$ is a $k$-cycle for some $k>4$, then there is a cycle $C^{\prime}$ formed by a path in $C$ and the edge $e$. Thus, $C$ would have a non-incident edge from the cycle $C^{\prime}$ so that $\tilde{G}$ would be homeomorphic to $H$.

Hence, all cycles in $\tilde{G}$ are 3 -cycles or 4-cycles. Suppose $C$ is a 3 -cycle with another cycle $C^{\prime}$ in $\tilde{G}$. Either $C$ and $C^{\prime}$ share an edge giving a longer cycle (contradicting the maximality of $C$ ) or $C^{\prime}$ has an edge non-incident to $C$. Hence, $C$ must be a 4 -cycle if $\tilde{G}$ has multiple cycles. If two 4 -cycles $C$ and $C^{\prime}$ only share a vertex or a single edge, then $C$ would have a non-incident edge in $C^{\prime}$. Hence, $C$ and $C^{\prime}$ must share two edges. If the two edges are non-incident, then $C_{1}$ and $C_{2}$ form a $K_{4}$. Thus, $\tilde{G}$ either forms a $K_{4}$ or all the 4 -cycles share a common path consisting of the two incident edges $(x, z)$ and $(y, z)$. Thus, all 3-cycles have the common edge $(x, y)$ if it exists. Any non-cycle edge $e$ must be incident to all the cycles implying that $e$ is either $(x, z)$ or $(y, z)$ for some vertex $z$ of degree 1 . Thus, if $\tilde{G}$ has multiple cycles but is not a $K_{4}$, then $\tilde{G}$ is a subgraph of some $K_{3}$-multiedge. Finally, if $C$ is the only cycle, then all the vertices not in $C$ have degree 1 so that $\tilde{G}$ is a circular caterpillar.

Together Corollary 9 and Lemma 10 allow us to determine when a graph always has a SEFE with any planar graph with the following lemma:

Lemma 11 A graph $G$ has a SEFE with any planar graph if only if $G \in \mathcal{P}_{\text {SEFE }}$.

Proof. We prove necessity by showing that each $G_{1} \in \mathcal{P} \backslash \mathcal{P}_{\text {SEFE }}$ does not have a SEFE with every $G_{2} \in \mathcal{P}$. In all the 17 pairs of Theorem 8, both graphs have a subgraph homeomorphic to $G_{1,1}$ that is a $K_{3}$ and a disjoint edge; see Fig. 5(a). By Lemma 10, we know that that $G_{1}$ contains a subgraph homeomorphic to $G_{1,1}$. Thus, $\left(G_{1}, G_{2}\right)$ cannot have a SEFE by Corollary 9 in which $G_{2}$ contains a subgraph homeomorphic to $G_{1,2} \in \mathcal{P}$.

To show sufficiency, we must show that every graph in $G \in \mathcal{P}_{\text {SEFE }}$ has a SEFE. We do this by showing how to efficiently compute a SEFE for the class of graphs in $\mathcal{P}_{\text {SEFE. Frati }}$ [8] gave an algorithm that finds a SEFE for forests and planar graphs without explicitly bounding the number of bends per edge. Our algorithm computes a SEFE by drawing each edge with a modification of the optimal Euclidean shortest path algorithm that runs in $O(n \log n)$ time [11]. The modification is to determine the shortest path among a set of line segments (that do not intersect except at endpoints) in the plane in which at least a distance (of arbitrarily small) $\varepsilon$ is always left between the path and the endpoint of any segment. This can be done using Minkowski sums such that the minimum distance from each endpoint is $2^{n / i} \varepsilon$ in step $i$ for $i \in[1 . . n]$.

For each step $i$, a new bend $b_{i, k}$ is either caused by an endpoint $p_{k}$ of an edge or a bend $b_{j, k}$ from a previous step $2 \leq j<i$. However, for each such bend $b_{i, k}$ only at most two points in the set $\left\{p_{k}, b_{2, k}, \ldots b_{i-1, k}\right\}$ (the inner and outer ones) contribute - bends added more recently hide bends caused by the original point $p_{k}$ in previous steps. Hence, each time we add edges, at most $O(n)$ new bends are being introduced. Since the size of the vertex set grows by $O(n)$ for each step, this gives an overall running time of $\sum_{i=1}^{n} O(i \cdot n \log i \cdot n)=O\left(n^{2} \log n\right)$. 
Let $G_{1} \in \mathcal{P}_{\mathrm{SEFE}}$ and $G_{2} \in \mathcal{P}$. First, we draw $G_{2}$ in $O(n)$ time. We then find an embedding of $G_{2}$ and draw $G_{2}$ on an $(n-2) \times(n-2)$ grid, both done in $O(n)$ time $[3,5]$. Some of the edges of $G_{1}$ were drawn with $G_{2}$. We can ignore the edges in $G_{2} \backslash G_{1}$ as we draw the rest of $G_{1}$. For a forest or a circular caterpillar in which the cycle has not yet been drawn, there is a single face giving a shortest Euclidean path between any two vertices. For a circular caterpillar with the cycle already drawn, the remaining points either lie inside or outside of the cycle. All edges are incident to the cycle. Hence, a Euclidean path always exists from vertices of the cycle to vertices of degree 1 . For a graph with multiple cycles, it is a $K_{4}$ or a subgraph of a $K_{3}$-multiedge with a 4-cycle $C$ that has two vertices $x$ and $y$ of degree greater than 2. We finish drawing $C$. For $K_{4}$, one chord is drawn inside of $C$, while the other chord is drawn outside of $C$. For a $K_{3}$-multiedge, any path from $x$ to $y$ is either the edge $(x, y)$ or the path $x \rightsquigarrow z \rightsquigarrow y$ from some degree-2 vertex $z$. The edge $(x, y)$ can drawn inside of $C$ to start. For the other paths, there must always exist Euclidean paths from $x$ and $y$ to the common vertex $z$ that lies inside some cycle drawn so far. Any remaining edges must be incident to $x$ or $y$ in which a Euclidean path must also exist.

Lemmas 10 and 11 together imply the following characterization:

Theorem 12 The following two statements are equivalent: A graph has a SEFE with any planar graph if only if

- it does not contain a subgraph homeomorphic to a $K_{3}$ and a disjoint edge.

- it is either (i) a forest, (ii) a circular caterpillar, (iii) a $K_{4}$, or (iv) a subgraph of a $K_{3}$-multiedge.

\section{Characterizing SEFE with Outerplanar Graphs}

We next determine which biconnected outerplanar graphs always have a SEFE with any other outerplanar graph. A $K_{3}$-cycle is an $n$-cycle $C$ with chords such that every chord forms a 3 -cycle with edges from $C$; see Fig. 2(e).

The following lemma provides an analogous result for biconnected outerplanar graphs with respect to the outerplanar graphs $\mathcal{O}$ that Lemma 10 does for the planar graphs $\mathcal{P}$. The omitted proof can be found in [7]. The set $\mathcal{O}_{\text {SEFE }}$ of $K_{3}$-cycles is shown to be the set of biconnected outerplanar graphs that do not contain $\left(G_{14,1}, G_{14,2}\right)$ as a fixed edge minor pair. This is the only pair of Theorem 8 in which both graphs are biconnected and outerplanar. The graphs $G_{14,1}$ and $G_{14,2}$ are both isomorphic to a 6 -cycle with a chord that forms two 4-cycles.

Lemma $13 G$ is in $\mathcal{O}_{\text {SEFE }}$ if and only if $G$ does not contain a subgraph homeomorphic to $G_{14,1}$.

The omitted proof of the following lemma also appears in [7]. The key idea is to use Euclidean shortest paths again to draw each edge that is not in the intersection. Special care is taken for pairs of edges $(x, z)$ and $(y, z)$ when the chord $(x, y)$ is in the intersection. First, the edge $(y, z)$ is routed to $x$ and then both edges proceed within a small distance of each other from vertex $z$. Remaining chords can always be drawn inside the outerface of the $K_{3}$-cycle since each has a degree- 2 vertex $z$ on the outerface that is adjacent to both endpoints. 


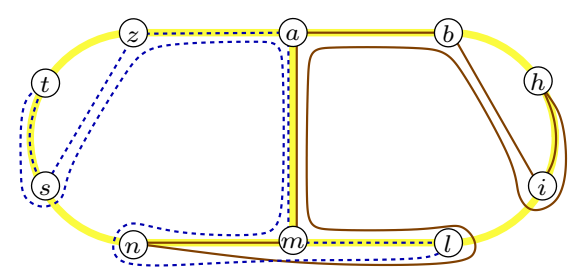

(a)

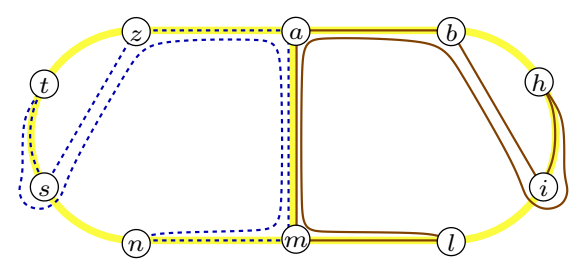

(b)

Fig. 8. Two biconnected outerplanar graphs with a common chord $(a, m)$ do not have a SEFE in (a) given that $(a, m)$ and its adjacent endpoints match the forbidden labeling of $\left(G_{14,1}, G_{14,2}\right)$. The same pair in (b) has a SEFE since this is not the case.

Lemma 14 A biconnected outerplanar graph $G$ has a SEFE with any outerplanar graph if only if $G \in \mathcal{O}_{\text {SEFE }}$.

Lemmas 13 and 14 together give the following characterization:

Theorem 15 The following two statements are equivalent: A biconnected outerplanar graph has a SEFE with any outerplanar graph if only if

- it does not contain a subgraph homeomorphic to $G_{14,1}$.

- it is a $K_{3}$-cycle.

\section{Deciding SEFE for Biconnected Outerplanar Graphs}

Corollary 9 provided a necessary but insufficient condition for the SEFE of two planar graphs. However, for the restricted case of two biconnected outerplanar graphs, we can give a necessary and sufficient condition.

Lemma 16 The biconnected outerplanar graph pair $\left(G_{1}, G_{2}\right)$ has a SEFE if and only if $G_{1}$ and $G_{2}$ does not have the fixed edge minor pair $\left(G_{14,1}, G_{14,2}\right)$.

The omitted proof found in [7] compares the labelings of the two outerfaces and the chords in the intersection to see if they match the forbidden labeling of the outerplanar graphs of $\left(G_{14,1}, G_{14,2}\right)$; see Fig. 8. If so, the pair does not have a SEFE. Otherwise, an algorithm that runs in $O\left(n^{2} \log n\right)$ time is given to produce a SEFE in which the cycles involving common chords in each graph are closed in such a way as to avoid any crossings.

Theorem 17 Deciding whether a pair of biconnected outerplanar graphs $\left(G_{1}, G_{2}\right)$ has a SEFE can be done in $O(n)$ time.

The omitted proof found in [7] uses the conditions on the common chords in the intersection in the proof of Lemma 16. This condition can be checked in linear time, which yields a linear-time decision algorithm.

\section{Conclusion}

We gave a necessary condition for whether two graphs can have a SEFE in terms of 17 fixed edge minor pairs. This allowed us to characterize the graphs that always have a SEFE with any planar graph. We also characterized the class of biconnected outerplanar graphs that have a SEFE with any outerplanar graph. 
For the restricted case of two biconnected outerplanar graphs, deciding whether they have a SEFE can be done in linear-time.

While our results may be helpful in solving bigger open problems, there are still no known algorithms for testing whether a pair of planar graphs has a SEFE in polynomial time. Finding all fixed edge minor pairs of planar graphs would give a sufficient condition for their SEFE. This may lead to a polynomialtime decision algorithm, an improvement over the ILP crossing minimization algorithm in [4].

\section{References}

1. U. Brandes, C. Erten, J. Fowler, F. Frati, M. Geyer, C. Gutwenger, S. Hong, M. Kaufmann, S. Kobourov, G. Liotta, P. Mutzel, and A. Symvonis. Colored simultaneous geometric embeddings. In 13th Computing and Combinatorics Conference, COCOON 200\%, volume 4598, pages 254-263, 2007.

2. P. Brass, E. Cenek, C. A. Duncan, A. Efrat, C. Erten, D. Ismailescu, S. G. Kobourov, A. Lubiw, and J. S. B. Mitchell. On simultaneous graph embedding. Computational Geometry, 36(2):117-130, 2007.

3. N. Chiba, T. Nishizeki, S. Abe, and T. Ozawa. A linear algorithm for embedding planar graphs using PQ-trees. J. Comput. Syst. Sci., 30(1):54-76, 1985.

4. M. Chimani, M. Jünger, and M. Schulz. Crossing minimization meets simultaneous drawing. In IEEE Pacific Visualization Symposium 2008, pages 33-40, 2008.

5. H. de Fraysseix, J. Pach, and R. Pollack. How to draw a planar graph on a grid. Combinatorica, 10(1):41-51, 1990.

6. A. Estrella-Balderrama, E. Gassner, M. Jünger, M. Percan, M. Schaefer, and M. Schulz. Simultaneous geometric graph embeddings. In 15th Symposium on Graph Drawing, GD 200\%, pages 280-290, 2008.

7. J. J. Fowler, M. Jünger, S. G. Kobourov, and M. Schulz. Characterizations of restricted pairs of planar graphs allowing simultaneous embedding with fixed edges. Technical Report TR08-01, University of Arizona, 2008. ftp://ftp.cs.arizona.edu/reports/2008/TR08-01.pdf.

8. F. Frati. Embedding graphs simultaneously with fixed edges. In 14th Symposium on Graph Drawing, GD 2006, pages 108-113, 2007.

9. E. Gassner, M. Jünger, M. Percan, M. Schaefer, and M. Schulz. Simultaneous graph embeddings with fixed edges. In 32nd International Workshop on Graph-Theoretic Concepts in Computer Science, WG 2006, pages 325-335, 2006.

10. M. Geyer, M. Kaufmann, and I. Vrto. Two trees which are self-intersecting when drawn simultaneously. In 13th Symposium on Graph Drawing, GD 2005, pages 201-210, 2006.

11. J. Hershberger and S. Suri. An optimal algorithm for Euclidean shortest paths in the plane. SIAM Journal on Computing, 28(6):2215-2256, 1999.

12. C. Kuratowski. Sur les problèmes des courbes gauches en Topologie. Fund. Math., 15:271-283, 1930.

13. J. Pach and R. Wenger. Embedding planar graphs at fixed vertex locations. In Proceedings of the 6th Symposium on Graph Drawing, GD 1998, pages 263-274, 1998.

14. K. Wagner. Über eine Eigenschaft der ebenen Komplexe. Math. Ann., 114(1):570590, 1937. 